\title{
1 In Silico Processing of the Complete CRISPR-Cas Spacer Space for \\ 2 Identification of PAM Sequences
}

3

$4 \quad$ Brian J Mendoza ${ }^{1}$

5 Cong $\mathrm{T}$ Trinh $^{1,2}$

6

$7 \quad{ }^{1}$ Department of Chemical and Biomolecular Engineering, University of Tennessee, Knoxville

8 TN, USA

9

10 Correspondence: Dr. Cong $\mathrm{T}$ Trinh, Department of Chemical and Biomolecular

11 Engineering, University of Tennessee, 1512 Middle Drive, 37996 Knoxville, TN, USA

12

$13 \quad$ E-mail: ctrinh@utk.edu

14

15 Keywords: PAM, protospacer, spacerome, CRISPR, Cas, CASPER, CASPERpam

16

17 


\section{Abstract}

19 Despite extensive exploration of the diversity of CRISPR (Clustered Regularly Interspaced

20 Short Palindromic Repeats) systems, biological applications have been mostly confined to

21 Class 2 systems, specifically the Cas9 and Cas12 (formerly Cpf1) single effector proteins. A

22 key limitation of exploring and utilizing other CRISPR-Cas systems with unique

23 functionalities, particularly Class I types and their multi-protein effector complex, is the

24 knowledge of the system's protospacer adjacent motif (PAM) sequence identity. In this work,

25 we developed a systematic pipeline, named CASPERpam, that enables us to comprehensively

26 assess the PAM sequences of all the available CRISPR-Cas systems in the NCBI database of

27 bacterial genomes. The CASPERpam analysis revealed that within the 30,389 assemblies

28 previously screen for CRISPR arrays, there exists 26,364 spacers that match somewhere in

29 the viral, bacterial, and plasmid databases of NCBI, using the constraints of $95 \%$ sequence

30 identity and $95 \%$ sequence coverage for blast hits. When grouping these results by species,

31 we were able to identify putative PAM sequences for 1,049 among 1,493 unique species.

32 The remaining species either have insufficient data or an undetermined result from the

33 analysis. Finally, we were able to infer certain design principles that are relevant for

34 understanding PAM diversity and a baseline for further experimental studies including PAM

35 assays. We envision CASPERpam is a useful bioinformatic tool for understanding and

36 harnessing the diversity of CRISPR systems. 


\section{1. Introduction}

39 The bacterial adaptive immunity system CRISPR (Clustered Regularly Interspaced Short

40 Palindromic Repeats), is near-ubiquitous across the archaea and bacterial kingdoms and

41 exhibits extensive diversity [1]. Despite this heterogeneity, there exists two fundamental

42 biochemical behaviors shared among all CRISPR systems: adaptation and interference [2].

43 The first stage, adaptation, is relatively conserved across species and involves the process by

44 which foreign DNA (or RNA) is acquired and inserted into the CRISPR array. The second

45 step, interference, involves the expression of the acquired sequences of the array and

46 processing them into short RNAs for recognition and cleavage of the foreign DNA/RNA. To

47 avoid self-cleavage, a short sequence known as a protospacer adjacent motif or PAM, appears

48 just outside the recognition sequence. The PAM serves as a checkpoint to ensure the correct

49 target has been found [3-5]. This first step is critical to the interference step, as even a

50 perfectly matched guide RNA sequence without the proper PAM sequence will fail to

51 achieve any activity $[4,6]$.

52 Significant research has been devoted to the classification of CRISPR-Cas systems to

53 help define common functionalities [1, 7]. The current system contains two main classes,

54 distinguished by a single (Class 2) or multi (Class 1) protein effector complex and further

55 classified into 6 types and 22 subtypes [1]. Experimental studies have shown however that

56 PAM sequences are particularly diverse and may vary from species to species even within

57 subtypes [8]. The knowledge of the PAM sequence is thus critical for harnessing CRISPR-

58 Cas systems, and the current classification system offers incomplete insight into a species'

59 PAM sequence. As research increasingly branches out from the canonical Cas9 of

60 Streptococcus pyogenes and its Type II relatives to discover novel functionalities in alternate

61 CRISPR-Cas systems, the ability to study these systems will be limited to the speed in which

62 PAM sequences can be determined $[9,10]$. 
63 Three common methods exist for determining the PAM sequence and have been used 64 to varying degrees of efficacy: in vivo selection [11-13], in vitro assays [13, 14], and in silico 65 analysis $[4,15,16]$. The first two methods are experimentally driven and provide the most 66 accurate determination of the PAM sequence. However, they require time-intensive 67 experimental procedures, and rely on effective molecular biology tools suited to the organism 68 in question to be previously developed (in vivo), or the ability to clone out and express an 69 active system heterologously (in vitro) [5]. In contrast, the in silico method uses sequence 70 similarity of existing spacers in an organism's genome to that of invasive genetic elements to 71 suggest a potential PAM sequence [15]. While the method is less accurate and complete than 72 that of its experimental counterparts, it requires simply a query of CRISPR array(s) against 73 potential target genomes, which are readily available in ever-expanding sequencing 74 databases. Although this method has been performed for a number of organisms to elucidate 75 potential PAM sequences, a comprehensive PAM analysis of all available CRISPR-carrying 76 organisms has not previously been performed.

77 We have recently presented the CRISPR Associated Software for Pathway 78 Engineering and Research (CASPER) for enhanced on- and off-target prediction of gRNA 79 designs and multi-targeting analysis for single and consortium of organisms using various 80 Cas enzymes [17]. In this study, we have developed the CASPERpam algorithm to 81 systematically and comprehensively perform a complete PAM analysis for all prokaryotes in 82 the NCBI database for which sufficient data exists to investigate potential target matches. 83 From the generated PAM sequence database, we were able to infer certain design principles 84 that are relevant for understanding PAM diversity and provide a baseline for further 85 experimental studies including PAM assays. The PAM sequence database and categories 86 have been integrated into the CASPER platform as a new feature. We anticipate the 87 CASPERpam tool will enable further research and engineering of prokaryotes using their 
88 native CRISPR machinery and aid in the development of novel CRISPR-Cas systems for

89 heterologous expression in eukaryotes.

\section{2. Methods}

92 This section presents the CASPERpam algorithm for processing the complete CRISPR-Cas 93 spacer space to identify PAM sequences to complement the resources of CASPER [17] for

94 research in novel CRISPR-Cas systems. The CASPERpam algorithm contains 5 steps for 95 spacer collection, genome collection, and PAM detection and analysis (Figure 1).

\section{2.1. Spacer collection for PAM analysis}

98 Based on the 720,391 spacers detected across 42,352 CRISPR arrays from 30,389 assemblies 99 collected and analyzed by Shmakov et al. [18], a total of 26,364 hits were detected by a 100 BLASTN search across the viral (13,885 assemblies), plasmid (11,218 assemblies), and 101 bacterial/prokaryotic (12,9209 assemblies) genome databases of NCBI, using the $95 \%$ 102 sequence identity and 95\% coverage constraints to gather only hits with sufficient similarity 103 (Step 1, Figure 1). There were no spacers that generated multiple hits. To identify a PAM 104 sequence, we collected the hit sequence and included an additional 10 nucleotides on either 105 side (the "collected" sequence). Because the hit sequence itself may only be a partial match to 106 the spacer, we extrapolated endpoints of the spacer within the collected sequence.

107 Specifically, the spacer and collected sequences were aligned by finding the largest perfectly 108 matched sequence shared between the two sequences. Then the indices of the spacer 109 sequence's start and end within the collected sequence were used to define the locations of 110 the flanking sequences.

111

\section{2.2. Genome collection for PAM analysis}


113 From the spacer collection suitable for PAM analysis, we could narrow the number of

114 assemblies from 30,389 to 6,955 (Step 2, Figure 1). Since multiple assemblies can belong to

115 the same species, we clustered them at the species level to create a total of 1,493 species

116 (Step 3, Figure 1). By choosing species with at least 2 spacer hits, we further reduced the

117 number of species from 1,493 to 1,049 for which we could search for PAMs (Step 4, Figure

118 1). In our analysis, strains and substrains were grouped together under a common species.

119 While in some rare cases it is possible that PAM sequences might differ between strains and 120 substrains due to random mutation or perhaps horizontal gene transfer of CRISPR-Cas 121 machinery among strains [7], we would not have been able to perform further analysis in a 122 vast majority of species due to the limited number of hits belonging to each substrain.

\section{$124 \quad$ 2.3. PAM detection and analysis}

125 The Cas type was used to determine whether a PAM was located 5' (upstream) or 3' 126 (downstream) of the hit (Step 5, Figure 1). For type I, III (rPAM), IV, and V systems, the 127 PAM has been either shown or inferred to occur 5' of the spacer sequence [19-21]. Type II 128 systems, however have 3' PAMs of the spacer sequence. Each species was assigned a 129 CRISPR-Cas system based on the data provided by Makarova et al. [1,7]. For those species 130 that did not appear in the prior classification studies, the genus was used to match the species 131 to a relative and its Cas type was inferred from the type of its relative.

132 To obtain the PAM for each of the 1,049 species, bitwise scoring [22] was used to 133 determine the important positions (Step 5, Figure 1). Positions with an R-score higher than a 134 half a standard deviation above the mean were considered significant. As an additional 135 constraint, the R-scores for positions farther than 5 nucleotides away from the spacer 136 sequence were subject to the more stringent requirement of full standard deviation above the 137 mean to further filter out noise from positions unlikely to contribute to the PAM sequence. 
138 Within each significant position, if a nucleotide had a relative frequency higher than 0.5 , it

139 was considered to be the consensus nucleotide. If no nucleotide was higher than 0.5 , the

140 nucleotide(s) that had relative frequency greater than 0.25 were considered to be part of the

141 PAM.

142 The R tool ggseqlogo was used to create a bitwise graph for each species to determine

143 the PAM from the appropriate flanking sequence (5' or 3') [22]. In many cases, sequences

144 obtained were shorter than 10 nucleotides due to the spacer index starting in the flanking

145 sequence and/or appearing at the end of an assembly or near an ambiguous sequence (set of

146 'N's). To satisfy the symmetrical sequence length requirement of ggseqlogo, flanking

147 sequences smaller than 6 were discarded and the remaining were truncated to the length of

148 the smallest sequence.

\section{2.4. Computation}

151 NCBI servers were used solely for query purposes (https://www.ncbi.nlm.nih.gov). All 152 genomes needed to identify flanking sequences of the spacer hits were downloaded from 153 NCBI (RefSeq/GenBank) onto an external hard drive (as of 11/24/2017). Python (ver. 3.6)

154 was used to process the data and sequences. R (ver. 3.4.3) statistical programming language 155 was used to run ggseqlogo and generate the sequence bitwise plots [22]. All computational 156 work was performed on a Macintosh MacBook Pro computer (Apple Inc., 2013). Code is 157 available at https://github.com/TrinhLab/CASPERpam.

\section{3. Results and Discussion}

\section{3.1. Predictive PAM sequences match experimental evidence}

161 To validate the CASPERpam algorithm, we compared the predicted and experimentally

162 derived PAM sequences for a set of 12 species spanning both CRISPR classes and multiple 
163 types/subtypes (Figure 2). Our result shows that CASPERpam could generate the exact match

164 (green boxes) for the experimentally derived PAM sequences in 3 of the 12 species including

165 P. aeruginosa (Figure 2A), Acinetobacter baumannii (Figure 2B), and E. coli (Figure 2C). In

166 addition, we found strong correlation (blue boxes) between the experimental and predicted

167 PAM sequences of 4 species: S. pyogenes (Figure 2D), C. difficile (Figure 2E), L. bacterium

168 (Figure 2F), and B. halodurans (Figure 2G). An additional two species had weak correlation

169 (yellow boxes): S. thermophilus (Figure 2H) and T. denticola (Figure 2I). Weak correlation

170 species are defined here as partial matches where half or less of the predicted PAM matched

171 with the experimentally derived sequence. Finally, we did not find a match (red boxes) for

172 the 3 remaining species: N. meningitidis (Figure 2J), C. jejuni (Figure 2K), and S. aureus

173 (Figure 2L).

174 In our analysis, we observed that the complete and strong partial matches appeared in

175 species that have relatively high spacer hits and consensus Cas-types (Supplementary Table

$176 \mathrm{~S} 1)$. In contrast, the non-matches correlated to a smaller number of hits. A notable exception

177 is Bacillus halodurans, which despite a relatively low number of hits returned a strong match

178 to its experimental PAM. (Table 1). Interestingly, type II species seem particularly prone to

179 inaccurate predictions of the PAM sequence, despite large amounts of hits and a consensus

180 Cas-type in certain scenarios. In contrast to type I systems where the PAM sequence may be

181 attributed to either the acquisition or interference stage, type II interference mechanisms have

182 been shown to depend heavily on an accurate PAM sequence [6, 12-14]. Because sequencing

183 results belong to existing phages and other mobile genetic elements, i.e. those that have

184 survived and escaped CRISPR-Cas interference, they may have acquired favorable mutations

185 in the PAM identification region, contributing to an incorrect PAM sequence being derived 186 by CASPERpam. 


\section{$192 \quad 3.2$ Quantification of the quality of in silico PAM prediction}

193 To quantify the accuracy of PAM prediction for a given species, we developed a metric, the

194 PAM confidence score (PamCoS), to determine whether the information obtained from the 195 database (NCBI RefSeq) is of sufficient quantity and quality. PamCoS is calculated as 196 follows:

$$
\operatorname{PamCoS}=h \times\left(\frac{C_{m}}{C_{T}} \times \frac{1}{N_{S}}-H_{\text {avgdev }}\right)
$$

In equation 1, the value $h$ represents the number of hits from all the spacers (Supplementary

Table S1). $\mathrm{C}_{\mathrm{m}}$ and $\mathrm{C}_{\mathrm{T}}$ refer to the number of detected Cas arrays that match the consensus

$$
\begin{gathered}
H_{\text {avgdev }}=\frac{\sum H_{i, s i g}-H_{a v g}}{n_{s i g}} \\
H_{i}=-\sum f_{a, i} \times \log _{2} f_{a, i}
\end{gathered}
$$

209 In equation $2, \mathrm{H}_{\mathrm{i}, \mathrm{sig}}$ is the Shannon entropy of a significant position; $\mathrm{H}_{\mathrm{avg}}$ represents the 210 average Shannon entropy across all positions in the flanking sequence; and $\mathrm{n}_{\text {sig }}$ is the number 
211 of significant positions. The $f_{a, i}$ term in equation 3 is the relative frequency of a given 212 nucleotide "a" at position i.

213 We used PamCoS to score all 1049 species in the CASPERpam produced database 214 (Figure 1 and Supplementary Table S1). The species with the top 40 PamCoS scores are 215 presented in Table 1, along with a select number of additional species for which an 216 experimentally derived PAM sequence is available. The relative PamCoS score is shown in 217 this table, where the scores have been normalized (0 to 100) based on the highest PamCoS 218 score of Pseudomonas aeruginosa (2851.61) in the database. High PamCoS scores correlate 219 well with accurate predictions by CASPERpam of the species with experimentally derived 220 PAMs, with the exception of type II species. It does seem however that after a certain 221 PamCoS threshold, the prediction for type II species becomes effective. For instance, $S$. 222 agalactiae and S. suis, two species with type II-A CRISPR-Cas systems, have PamCoS 223 scores over 400 (Supplementary Table S1) and exhibit the NGG motif as their predicted 224 PAM, the same as their experimentally proven close relative $S$. pyogenes. This suggests that 225 a PamCoS score of 400 can be used as a minimum threshold to guarantee accurate PAM 226 prediction for all Cas types. While only 8 species in the NCBI RefSeq database currently 227 satisfy this criteria, further sequencing efforts of microbial genomes will aid in providing the 228 necessary information for accurate in silico PAM prediction.

229 To assign a more flexible cutoff for the currently available data and using the 230 PamCoS scores of species with known PAMs, a score above 10 (0.35 relative PamCoS to $P$. 231 aeruginosa) seemed a reasonable threshold for PAM prediction. This however is not a strict 232 cutoff as the prediction of the PAM for B. halodurans proves lower PamCoS scoring species 233 may still lead to reasonably accurate PAMs. Lower PamCoS rated species must be viewed 234 with some skepticism, however. Due to the smaller number of hits, even when accompanied 235 by relatively low Shannon entropy values, these predicted sequences are more sensitive to 
236 false assignment of significant positions and nucleotides. Further, false sequence noise may

237 be compounded by potential Cas type ambiguity, as a non-consensus Cas-type hit may be 238 incorporated into the PAM sequence prediction.

239 Overall, use of the PamCoS score enhances in silico PAM analysis. Notably, PamCoS

240 allows the PAM sequences produced by CASPERpam to be assessed for their relative

241 accuracy and aid in narrowing the search space of sequences for experimental validation. For

242 instance, species with low PamCoS scores should be validated with a comprehensive in vitro

243 or in vivo methodology, using the results of CASPERpam as supplemental validation of

244 experimental discovery. On the other hand, species with relatively high PamCoS scores may

245 allow the CASPERpam prediction to be supplemented with only minimal experimentation to

246 provide confirmation of the predicted sequence.

247 Based on CASPERpam analysis, the number of species with PamCoS scores over the

248 threshold value of 10 (221) represents a very small portion of the tens of thousands of

249 bacterial and archaeal species that have been sequenced. It does however represent an order

250 of magnitude increase in the number of CRISPR-Cas systems that have had a PAM sequence

251 predicted or validated. The result provides a starting point to harness the extensive diversity

252 present across the CRISPR-Cas space.

253 Of the 221 species above the threshold value, $78 \%$ had a Class 1 system, an 254 understudied relative to their more popular single effector Class 2 cousins. Even though the 255 Type I system appears to have a greater variety in their PAM sequences [23], only a select 256 number of nucleotide patterns of PAMs are commonly used. These select PAM motifs are not 257 confined to a single Cas type, rather they are spread across systems (Figure 3A). One 258 potential explanation is that the PAM motif of Class 1 multi-effector systems is likely subject 259 to some degree of horizontal gene transfer. Particularly, the Cas8/10 and Cas 1/2 proteins, 260 likely responsible for PAM recognition during the interference and acquisition steps 
261 respectively, may be traded within microbial communities, creating within a species hybrid

262 Class 1 systems with a clearly assigned Cas-type using a PAM largely associated with 263 another.

\subsection{PAM prediction highlights diversity and patterns within and between Cas types}

266 To analyze the PAM diversity across different Cas-types, we gathered all PAM motifs of the

267221 species above the threshold value and grouped them into families of closely associated

268 motifs. We identified common patterns and assigned 5' and 3' PAM sequence categories

269 and/or subcategories to these sets for 218 species (Figures 3B and 3C). Each

270 category/subcategory is defined by a consensus motif that captures the general pattern. Three

271 species from the original set of 221 species, including Proteus mirabilis, Bifidobacterium

272 longum, and the Eubacterium sp., were removed from the analysis because CASPERpam

273 could not assign either a consensus Cas-type or the PAM motif was clearly the result of

274 multiple Cas-types.

275 The 5' PAM sequences contain 8 categories (J, K, L, M, N, O, P, and Q) (Figure 3A,

276 3B). The K and Q categories represent $61 \%$ of PAMs located on the 5' end. The 'CC' PAM

277 of the Q category and 'TTC' PAM of K are heavily represented (Figure 3A, 3B). These

278 groups are disproportionately associated with types I-F and I-B respectively; however, they

279 are not exclusive and also appear in species with other Cas-types. The PAM sequence space

280 of the 170 species with reliable 5' PAMs (as defined by the confidence score) can be seen

281 mapped together in Figure 3A.

282 It is striking to observe that none of the Cas types nor subtypes map to a unique PAM

283 category, with the exception of a few types with less than 5 species represented in the sample

284 (e.g. Cas-III-B and Cas-I-A). This result supports the view that the current CRISPR-Cas

285 classification method is imperfect likely due to the CRISPR-Cas systems' similarity and 
286 modularity prone to horizontal gene transfer [1]. It also prevents a simple classification based 287 on sequence homology or phylogenetics from perfectly partitioning the heterogeneity of these 288 systems.

289 The 3' PAM sequences consists of 3 categories (X, Y, and Z) with the X category 290 broken into a further 4 subcategories (X1, X2, X3, X4) (Figure 3C). Among the 3' PAM 291 sequences, the category Y, with the NGG consensus motif, is quite common, representing $29225 \%$ of identified PAMs (Figures 3C, 3D). The category X, although representing two-thirds 293 of identified PAMs, has a much more broadly defined consensus motif $(\mathrm{N})_{\mathrm{n}} \mathrm{W}$ and lent itself 294 to subcategorization of which none reached a representation comparable to the Y category 295 (Figures 3C, 3D). The 'NGG' PAM found in the Y category is the most represented PAM 296 motif, but CASPERpam only identified two such species, with an exact 'NGG' PAM. Other 297 PAMs in the category were defined more broadly, with adenines and sometimes thymines 298 also highly represented at the 2 and 3 positions creating an NRR, and NKK PAMs. This 299 result is consistent with experimental evidence that type II-A systems such as the canonical $S$. 300 pyogenes exhibited some affinity for a 'NAG' PAM. Even though this detected promiscuity 301 may be overstated by the in silico analysis, it highlights the importance of future 302 experimental studies on species with a PAM falling in the Y category to focus on the relative 303 affinity for guanine over adenine or thymine.

$304 \quad$ Based on the 5' PAM space and the X and Z 3' PAM categories, we clearly observed 305 that there exists a high level of non-specificity in many PAM interacting domains. It cannot 306 be discounted that this diversity may be underrepresented by the in silico technique, as only 307 those with particularly effective targeting will appear across the targeted genomes [5]. On the 308 other hand, PAM sequences are likely to be mutated for phages to survive, and thus 309 sequencing assemblies may not represent the true form of the PAM sequence, but rather an 310 overrepresentation of a random set of mutated sequences despite a close spacer match. Such 
311 a hypothesis would result in the in silico process revealing greater non-specificity than would

312 be shown experimentally. Likely, these two effects are both present, and result in some

313 overly specific as well as some overly promiscuous sequence predictions from the in silico

314 method.

315

\section{4. Conclusion}

317 To harness a large, diverse class of alternative CRISPR systems outside of the group 318 of well studied Cas9 and Cas12 variants, it is critical to identify PAM sequences for 319 characterizing these novel CRISPR systems. Development of CASPERpam provides a 320 powerful tool to predict PAMs for a given species and allows subsequent experiments to 321 change from a determination to a validation study. While some previous studies have 322 performed such an in silico PAM analysis for a select number of species to guide 323 experimental studies of native CRISPR systems, this study has performed an exhaustive 324 PAM analysis for every CRISPR-Cas carrying bacterial and archaeal genome in the NCBI 325 database. As research moves into investigating a greater number of CRISPR-Cas systems 326 with novel functionalities, CASPERpam will be a useful bioinformatic tool for those seeking 327 to understand and harness the diversity of CRISPR systems.

\section{Abbreviations}

330 CRISPR, Clustered Regularly Interspaced Palindrome Repeats; CASPER, CRISPR 331 Associated Software for Pathway Engineering and Research; PAM, Protospacer Adjacent 332 Motif; PamCoS, PAM confidence score; NCBI, National Center for Biotechnology 333 Information. 
336 The research is funded by the DARPA YFA award \#D17AP00023. The views, opinions,

337 and/or findings contained in this article are those of the authors and should not be interpreted

338 as representing the official views or policies, either expressed or implied, of the funding 339 agencies.

340

\section{Conflict of Interest}

342 The authors declare no financial or commercial conflict of interest.

343 


\section{References}

345 1. Makarova, K.S., et al., An updated evolutionary classification of CRISPR-Cas

346 systems. Nat Rev Micro, 2015. 13(11): p. 722-736.

347 2. Barrangou, R., Diversity of CRISPR-Cas immune systems and molecular machines.

$348 \quad$ Genome Biol, 2015. 16: p. 247.

349 3. Shah, S.A., et al., Protospacer recognition motifs: Mixed identities and functional 350 diversity. RNA Biology, 2013. 10(5): p. 891-899.

351 4. Mojica, F.J.M., et al., Short motif sequences determine the targets of the prokaryotic 352 CRISPR defence system. Microbiology, 2009. 155(3): p. 733-740.

353 5. Leenay, R.T. and C.L. Beisel, Deciphering, Communicating, and Engineering the 354 CRISPR PAM. Journal of Molecular Biology, 2017. 429(2): p. 177-191.

355 6. Hsu, P.D., et al., DNA targeting specificity of RNA-guided Cas9 nucleases. Nat 356 Biotechnol, 2013. 31(9): p. 827-32.

357 7. Makarova, K.S. and E.V. Koonin, Annotation and Classification of CRISPR-Cas 358 Systems, in CRISPR: Methods and Protocols, M. Lundgren, E. Charpentier, and P.C. 359 Fineran, Editors. 2015, Springer New York: New York, NY. p. 47-75.

360 8. Cebrian-Serrano, A. and B. Davies, CRISPR-Cas orthologues and variants: 361 optimizing the repertoire, specificity and delivery of genome engineering tools. 362 Mamm Genome, 2017. 28(7-8): p. 247-261.

363 9. Horvath, P., et al., Diversity, activity, and evolution of CRISPR loci in Streptococcus 364 thermophilus. J Bacteriol, 2008. 190(4): p. 1401-12.

365 10. Ran, F.A., et al., In vivo genome editing using Staphylococcus aureus Cas9. Nature, 366 2015. 520(7546): p. 186-91.

367 11. Elmore, J., et al., DNA targeting by the type I-G and type I-A CRISPR-Cas systems of Pyrococcus furiosus. Nucleic Acids Research, 2015. 43(21): p. 10353-10363. 
369 12. Esvelt, K.M., et al., Orthogonal Cas9 proteins for RNA-guided gene regulation and $370 \quad$ editing. Nature methods, 2013. 10: p. 1116-21.

371 13. Zetsche, B., et al., Cpfl is a single RNA-guided endonuclease of a class 2 CRISPR372 Cas system. Cell, 2015. 163(3): p. 759-71.

373 14. Karvelis, T., et al., Rapid characterization of CRISPR-Cas9 protospacer adjacent 374 motif sequence elements. Genome Biology, 2015. 16: p. 253.

375 15. Biswas, A., et al., CRISPRTarget: Bioinformatic prediction and analysis of crRNA targets. RNA Biology, 2013. 10(5): p. 817-827.

16. Pyne, M.E., et al., Harnessing heterologous and endogenous CRISPR-Cas machineries for efficient markerless genome editing in Clostridium. 2016. 6: p. 25666.

380 17. Mendoza, B.J. and C.T. Trinh, Enhanced guide-RNA design and targeting analysis for precise CRISPR genome editing of single and consortia of industrially relevant

18. Shmakov, S.A., et al., The CRISPR Spacer Space Is Dominated by Sequences from Species-Specific Mobilomes. mBio, 2017. 8(5): p. e01397-17.

19. Xue, C., et al., CRISPR interference and priming varies with individual spacer sequences. Nucleic Acids Research, 2015. 43(22): p. 10831-10847.

20. Westra, Edze R., et al., CRISPR Immunity Relies on the Consecutive Binding and 388 Degradation of Negatively Supercoiled Invader DNA by Cascade and Cas3. Molecular Cell. 46(5): p. 595-605.

390 21. van der Oost, J., et al., Unravelling the structural and mechanistic basis of CRISPR391 Cas systems. Nature Reviews Microbiology, 2014. 12: p. 479.

392 22. Wagih, O., ggseqlogo: a versatile $R$ package for drawing sequence logos. 393 Bioinformatics, 2017. 33(22): p. 3645-3647. 
394 23. Fu, B.X., et al., High-Throughput Characterization of Cascade type I-E CRISPR

395 Guide Efficacy Reveals Unexpected PAM Diversity and Target Sequence Preferences.

396 Genetics, 2017. 206(4): p. 1727-1738.

397 24. Cady, K.C., et al., The CRISPR/Cas adaptive immune system of Pseudomonas 398 aeruginosa mediates resistance to naturally occurring and engineered phages. J $399 \quad$ Bacteriol, 2012. 194(21): p. 5728-38.

400 25. Karah, N., et al., CRISPR-cas Subtype I-Fb in Acinetobacter baumannii: Evolution 401 and Utilization for Strain Subtyping. PLOS ONE, 2015. 10(2): p. e0118205.

402 26. Luo, M.L., et al., Repurposing endogenous type I CRISPR-Cas systems for 403 programmable gene repression. Nucleic Acids Research, 2015. 43(1): p. 674-681.

404 27. Hou, Z., et al., Efficient genome engineering in human pluripotent stem cells using 405 Cas 9 from \&lt;em\&gt;Neisseria meningitidis\&lt;/em\&gt. Proceedings of the National 406 Academy of Sciences, 2013.110(39): p. 15644.

407 28. Boudry, P., et al., Function of the CRISPR-Cas System of the Human Pathogen 408 Clostridium difficile. MBio, 2015. 6(5): p. e01112-15.

409 29. DiCarlo, J.E., et al., Genome engineering in Saccharomyces cerevisiae using 410 CRISPR-Cas systems. Nucleic Acids Res, 2013. 41(7): p. 4336-43.

411 30. Kim, E., et al., In vivo genome editing with a small Cas9 orthologue derived from 412 Campylobacter jejuni. Nature Communications, 2017. 8: p. 14500.

413 31. Leenay, Ryan T., et al., Identifying and Visualizing Functional PAM Diversity across 414 CRISPR-Cas Systems. Molecular Cell, 2016. 62(1): p. 137-147. 
416 Table 1: List of select species with top PamCoS scores

\begin{tabular}{|c|c|c|c|c|c|c|c|}
\hline Genus & Species & Cas type & PAM Location & Exp. PAM & Consensus & $\begin{array}{l}\text { Relative } \\
\text { PamCoS }\end{array}$ & $\begin{array}{l}\text { Qualitative } \\
\text { assessment }\end{array}$ \\
\hline Pseudomonas & aeruginosa & CAS-I-F & $5^{\prime}$ & CC [24] & $\mathrm{CS}$ & 100.0000 & match \\
\hline Acinetobacter & baumannii & CAS-I-F & $5^{\prime}$ & $\mathrm{CC}[25]$ & $\mathrm{CC}$ & 59.3357 & match \\
\hline Salmonella & enterica & CAS-I-E & $5^{\prime}$ & - & ANG & 30.0805 & \\
\hline Streptococcus & agalactiae & CAS-II-A & $3^{\prime}$ & - & NGG & 24.6686 & \\
\hline Escherichia & coli & CAS-I-E & $5^{\prime}$ & AWG $[23,26]$ & ANS & 20.4289 & match \\
\hline Streptococcus & suis & CAS-II-A & $3^{\prime}$ & - & NGR & 16.7142 & \\
\hline Salinispora & arenicola & CAS-I-E & $5^{\prime}$ & - & SNNS & 16.1926 & \\
\hline Klebsiella & pneumoniae & CAS-I-E & $5^{\prime}$ & - & AWG & 14.3394 & \\
\hline Neisseria & meningitidis & CAS-II-C & $3^{\prime}$ & NNNNGATT $[12,27]$ & NNNNNMH & 13.2987 & no match \\
\hline Vibrio & cholerae & CAS-I-E & $5^{\prime}$ & - & $\mathrm{MM}$ & 9.4119 & \\
\hline Leptospira & interrogans & CAS-I-B & $5^{\prime}$ & - & ATG & 8.5312 & \\
\hline Listeria & monocytogenes & CAS-I-B & $5^{\prime}$ & - & $\mathrm{CCW}$ & 8.3762 & \\
\hline Clostridium & botulinum & CAS-III-B & $5^{\prime}$ & - & TNW & 8.2882 & \\
\hline Fusobacterium & nucleatum & CAS-I-B & $5^{\prime}$ & - & YNW & 8.2191 & \\
\hline Enterococcus & faecalis & CAS-II-A & $3^{\prime}$ & - & NGRNW & 7.2013 & \\
\hline Clostridium & difficile & CAS-I-B & $5^{\prime}$ & $\mathrm{CCW}$ [28] & WNNNW & 6.5629 & strong partial \\
\hline Salinispora & pacifica & CAS-I-E & $5^{\prime}$ & - & SNNS & 6.4865 & \\
\hline Pasteurella & multocida & CAS-I-F & $5^{\prime}$ & - & $\mathrm{CC}$ & 6.3487 & \\
\hline Moraxella & catarrhalis & CAS-I-F & $5^{\prime}$ & - & WNNY & 5.5702 & \\
\hline Leptospira & santarosai & CAS-I-E & $5^{\prime}$ & - & AAG & 5.5368 & \\
\hline Bifidobacterium & longum & CAS-II-C & $3^{\prime}$ & - & SNNS & 5.2296 & \\
\hline Serratia & marcescens & CAS-I-F & $5^{\prime}$ & - & AMS & 5.0828 & \\
\hline Clostridioides & difficile & CAS-I-B & $5^{\prime}$ & - & MNW & 4.4906 & \\
\hline Bifidobacterium & breve & CAS-I-C & $5^{\prime}$ & - & TTC & 3.9543 & \\
\hline Streptococcus & thermophilus & CAS-II-A & $3^{\prime}$ & NNNNGWWT $[9,12]$ & NNWNWA & 3.8955 & weak partial \\
\hline
\end{tabular}




\begin{tabular}{|c|c|c|c|c|c|c|c|}
\hline Methanosarcina & mazei & CAS-I-B & $5^{\prime}$ & - & TTA & 3.5871 & \\
\hline Streptococcus & pyogenes & CAS-II-A & $3^{\prime}$ & NGG [29] & NNR & 3.4889 & strong partial \\
\hline Yersinia & pseudotuberculosis & CAS-I-F & $5^{\prime}$ & - & $\mathrm{CC}$ & 3.4491 & \\
\hline Fusobacterium & necrophorum & CAS-II-A & $3^{\prime}$ & - & NGG & 3.3977 & \\
\hline Propionibacterium & freudenreichii & CAS-I-U & $5^{\prime}$ & - & SSAN & 3.0403 & \\
\hline Campylobacter & coli & CAS-I-B & $5^{\prime}$ & - & TNNNNNW & 2.9907 & \\
\hline Clostridium & novyi & CAS-I-B & $5^{\prime}$ & - & TNW & 2.8437 & \\
\hline Corynebacterium & ulcerans & CAS-I-E & $5^{\prime}$ & - & $\mathrm{AC}$ & 2.8430 & \\
\hline Salinispora & tropica & CAS-I-B & $5^{\prime}$ & - & SNNS & 2.8154 & \\
\hline Lactobacillus & paracasei & CAS-II-A & $3^{\prime}$ & - & TNNAA & 2.6750 & \\
\hline Aggregatibacter & actinomycet. & CAS-I-C & $5^{\prime}$ & - & $\mathrm{C}$ & 2.6473 & \\
\hline Treponema & pedis & CAS-II-C & $3^{\prime}$ & - & NNNWA & 2.6391 & \\
\hline Corynebacterium & striatum & CAS-I-E & $5^{\prime}$ & - & $\mathrm{AM}$ & 2.5033 & \\
\hline Lactobacillus & rhamnosus & CAS-II-A & $3^{\prime}$ & - & NNNWR & 2.4688 & \\
\hline Xenorhabdus & nematophila & CAS-I-E & $5^{\prime}$ & - & AAG & 2.4337 & \\
\hline Campylobacter & jejuni & CAS-II-C & $3^{\prime}$ & NNNNRYAC [30] & NNNNWNA & 1.5839 & no match \\
\hline Lachnospiraceae & bacterium & CAS-V-A & $3^{\prime}$ & TTTV [13] & $\mathrm{TNH}$ & 0.8278 & strong partial \\
\hline Treponema & denticola & CAS-II-A & $3^{\prime}$ & NAAAAN [12] & NNNWW & 0.6881 & weak partial \\
\hline Staphylococcus & aureus & CAS-III-A & $5^{\prime}$ & NNGRRT (3') & TTNNTNN & 0.2079 & no match \\
\hline Bacillus & halodurans & CAS-I-C & $5^{\prime}$ & YYC [31] & WNNTTN & 0.1347 & strong partial \\
\hline
\end{tabular}


bioRxiv preprint doi: https://doi.org/10.1101/274670; this version posted March 2 2018. The copyright holder for this preprint (which was not certified by peer review) is the author/funder, who has granted bioRxiv a license to display the preprint in perpetuity. It is made available under aCC-BY-NC-ND 4.0 International license.

419 Figure 1. CASPERpam algorithm for identifying PAM sequences

420

\section{Algorithms}

Step 1: Extract spacers

Step 2: Map spacers on to corresponding genomes

Step 3: Identify unique species with mapped spacers

Step 4: Filter species suitable for analysis and identify PAM sequences

Step 5: Perform PAM analysis and assess confidence
Results

30,389 genome assemblies

(720,391 spacers from 42,352 arrays)

6,955 genome assemblies with a match

(26,364 hits)

1,493 unique species represented

in the 6,955 genome assemblies

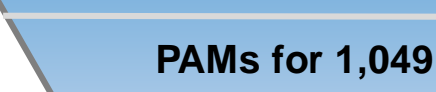

species with at least 2 hits

221 species

above

confidence

threshold 
bioRxiv preprint doi: https://doi.org/10.1101/274670; this version posted March 2,2018 . The copyright holder for this preprint (which was not certified by peer review) is the author/funder, who has granted bioRxiv a license to display the preprint in perpetuity. It is made available under aCC-BY-NC-ND 4.0 International license.

423 Figure 2. Predicted PAM sequences for experimentally validated species

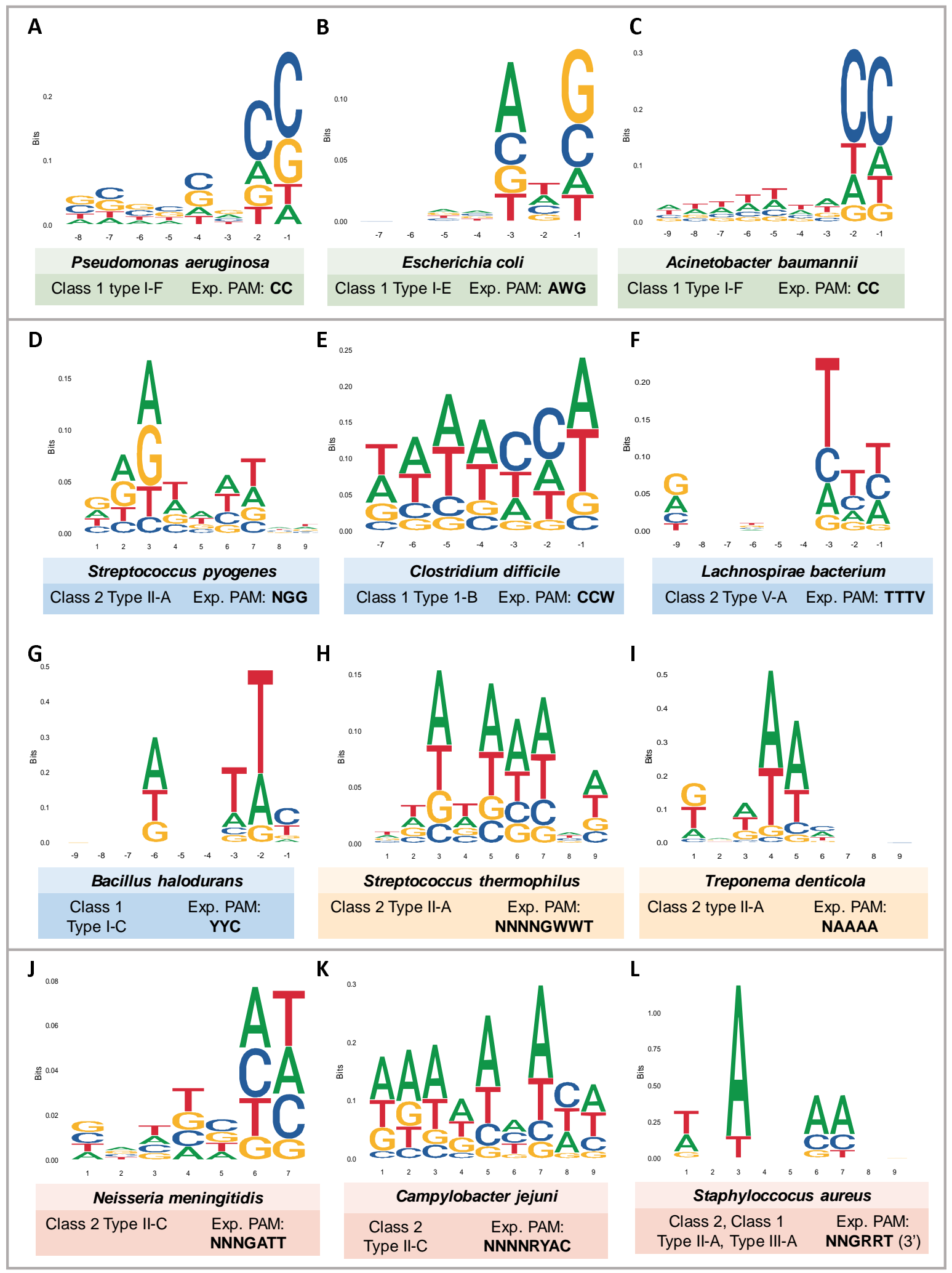

425 
bioRxiv preprint doi: https://doi.org/10.1101/274670; this version posted March 2, 2018. The copyright holder for this preprint (which was not certified by peer review) is the author/funder, who has granted bioRxiv a license to display the preprint in perpetuity. It is made available under aCC-BY-NC-ND 4.0 International license.

426 Figure 3. Predicted PAM sequences for select species

427

A

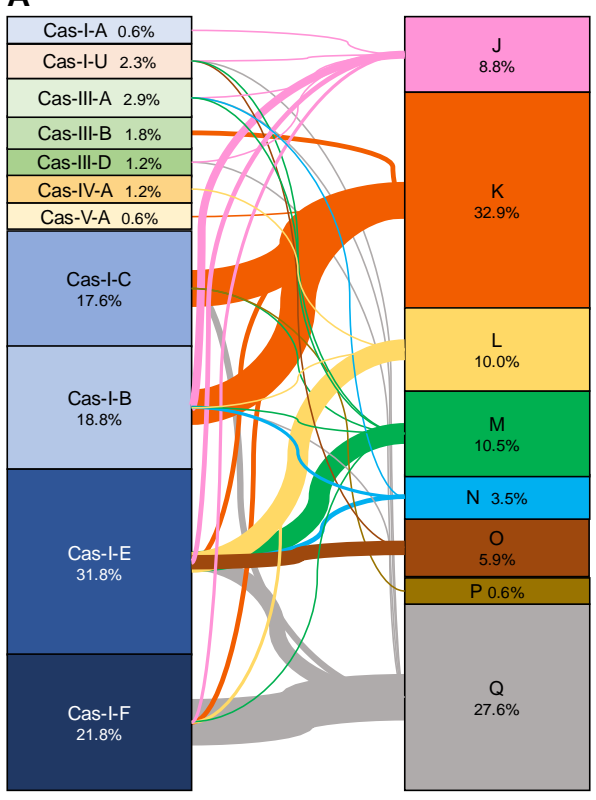

B

\begin{tabular}{|c|c|l|}
\hline Name & $\begin{array}{c}\text { Consensus } \\
\text { Motif }\end{array}$ & Common 5' PAM Sequences \\
\hline J & K,M(N) $)_{n>3}$ & W(N(N) $)_{n 5}(6)$ \\
\hline K & YYN & TTC (9), TTN (5), CCN (4), TNA (4) \\
\hline L & ANS,AAN & AAG (4), ANG (5) \\
\hline M & SNNS & SNNS (4), SNNSNNS (3) \\
\hline N & R(N) & \\
\hline O & AM & AC (2) \\
\hline P & HB & \\
\hline Q & CM,MC & CC $(20)$ \\
\hline
\end{tabular}

D

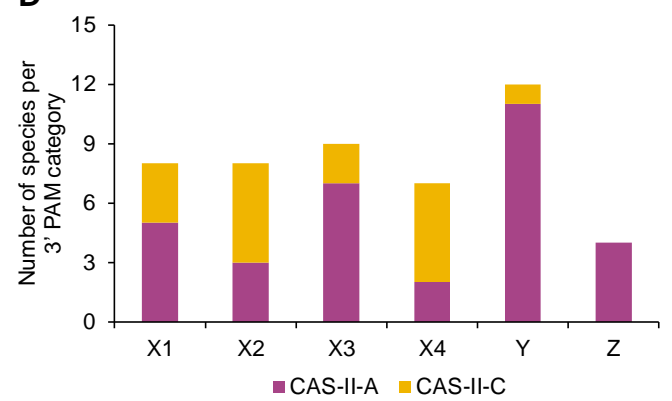

